Military Technical College Kobry El-Kobbah, Cairo, Egypt

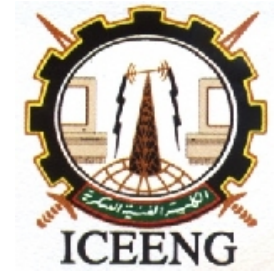

\section{$7^{\text {th }}$ International Conference on Electrical Engineering ICEENG 2010}

\title{
Multi-stages Visualized Parallel Beam CT Reconstruction with Performance Evaluation
}

\author{
By \\ Bassam Abd-Elwahab* \\ Mahmoud Gadallah** \\ Mohamed H. El-Mhalawy***
}

\section{Abstract:}

Computed tomography is a technique for estimating the interior of an object from measurements of radiation collected around the object. This radiation is projected either through or emitted from the object and the projections are reconstructed to form the object image. The reconstruction quality \& time depend on many factors such as: filteration domain, filter type and interpolation level. The objective of this paper is to develop an algorithm to simulate CT image reconstruction from projections (image simulated projections or raw data projections file). The developed program enables the user to investigate the effects of many factors mentioned above on the quality of the reconstructed images. The system has agraphical user interface (GUI) to facilitate changing the effictive factors and measuring the performance of the reconstruction. This system helps for studying the CT reconstruction process by introducing many factors selections for development as well as for training

\section{Keywords:}

CT reconstruction, CT reconstruction simulation, Filtered back-projection.

\footnotetext{
Egyptian Armed Forces

** Modern Academy, Cairo, Egypt

*** Egyptian Armed Forces
} 


\section{Introduction:}

In X-ray CT the contrast between one tissue type and another is associated with the different attenuation coefficients of the materials involved. The projections data taken at different views are the basis for tomographic image reconstruction. The goal of CT imaging is to derive the unknown attenuation coefficients based on the set of projections data.

In standard X-ray-based CT practice, a radiation source scans linearly along an object at a given view angle $\theta$, and after completion of the scan, the source and detector pair rotate a small angle $\theta$ and repeat the linear scan. This process continues until the completion of $180^{\circ}$ rotation. Thus for a parallel-beam data collection scheme, a total of $180^{\circ} \theta$ scans with angular displacement of $\theta$ is collected for a scan angle of 180. The need for a practical method of reconstruction gave rise to the filtered back-projection technique. The idea was first proposed by Bracewell [1] and later independently by Ramachandran \& Lakshiminyaranan [2].The technique is comprised of three sequential stages: filtering projections followed by back projecting the filtered projections and then image summation. Here these three steps are made sequentially; each view position is independently processed and displayed. It is important to build a graphical user interface program simulating the reconstruction process; this program is easily interfaced with multiple selections and controls. It gives the user options and facilities that enable studying, selecting, comparing and testing the algorithm. Different controls, multiple displays and frames enables the tracing of data progress through the algorithm form the input acquired projections until the output (reconstructed) image display.

\section{Mathematical Background:}

The mathematical basis for tomographic imaging has been described in a host of other publications [4] [5] [6] and a brief overview will be given here that is relevant for later descriptions of the algorithm used in the program.

The mathematical foundation of computed tomography was first derived by Radon [7] and published in 1917. However, it was not until the development of modern computers that the technique became at all viable. The original breakthrough was made by G. N. Hounsfield [8] of EMI Laboratories in 1972, showing a practical method for generating cross-sectional images of the head. Allan M Cormack of Tufts University independently invented the same process in two papers in the Journal of Applied Physics in 1963 and 1964 and later developed in [9].

\section{Radon Transform}

The projection of an object at a given angle $\theta$ is made up of a set of line integrals. In $\mathrm{X}$-ray $\mathrm{CT}$, the line integral represents the total attenuation of the beam of $\mathrm{x}$-rays as it travels in a straight line through the object. The resulting image is a $2 \mathrm{D}$ (or 3D) model of the attenuation coefficient. That is, we wish to find the image $\mu(x, y)$. The 
simplest and easiest to visualize method of scanning is the system of parallel projection, as used in the first scanners. For this discussion we consider the data to be collected as a series of parallel rays, at position $r$, across a projection at angle $\theta$. (see Figure 1). This is repeated for various angles. Attenuation occurs exponentially in tissue:

$$
\mathbf{I}=\mathbf{I}_{0} \mathbf{e}^{\int \mu(\tau) \mathrm{d} s}
$$

Where $\mu(-)$ is the attenuation coefficient at position a along the ray path $\mathrm{s}$. Therefore generally the total attenuation of a ray at position $r$, on the projection at angle $\theta$, is given by the line integral:

$$
p(r, \theta)=\ln \left(\frac{I}{I_{0}}\right)=\int \mu(x, y) d s
$$

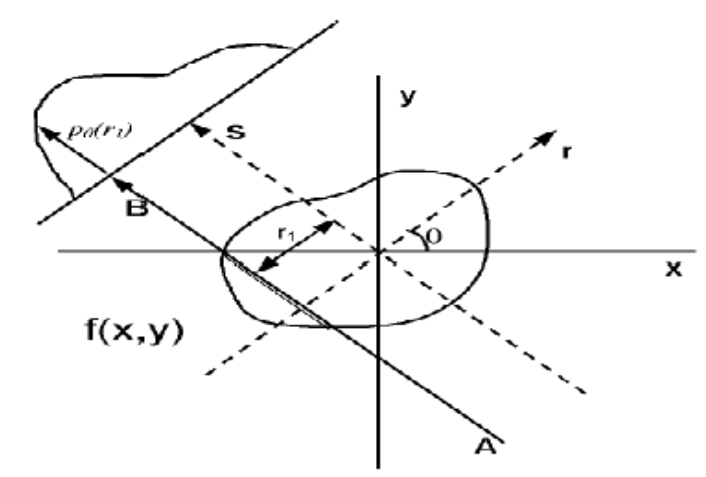

Figure (1):Parallel beam. Each projection is made up of the set of line integrals through the object.

Using the coordinate system of figure(1), the value of $r$ onto which the point $(x, y)$ will be projected at angle $\theta$ is given by

$$
\mathrm{x} \cos \theta+\mathrm{y}_{-} \operatorname{in} \theta=\mathrm{r}
$$

So equation 2 can be rewritten as

$$
p(r, \theta)=\iint_{-\infty}^{-\infty} f(x, y) \delta(x \cos \theta+\tau \sin \theta-r) d x d y
$$

Where $f(x, y)$ represents $\mu(x, y)$. This function is known as the Radon transform (or 'sinogram') of the 2D object, which tells us that if we had an in infinite number of one-dimensional projections of an object taken at an infinite number of angles, we could perfectly reconstruct the original object, $f(x, y)$. So to get $f(x, y)$ back, from the above equation means finding the inverse Radon transform.

The key to reconstruction of the projections is the Central Section Theorem (also called the Projection Theorem or Fourier Slice Theorem), which relates the measured projection data to the two-dimensional Fourier transform of the object cross section. 


\section{Filtered Back Projection}

In back projection the measurements at each projection are projected or 'smeared' back along the same line (same $\theta$ ), as in figures 2 and 3.

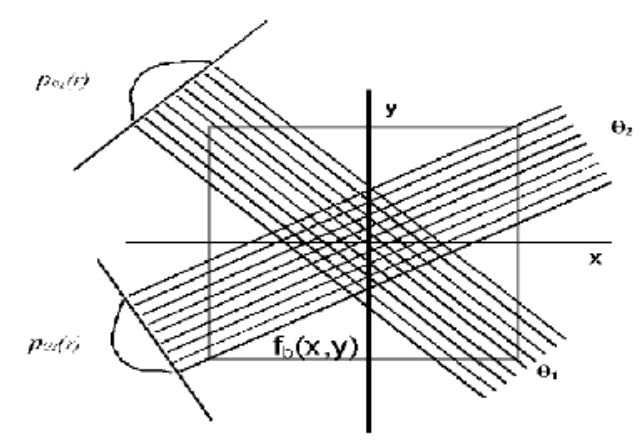

Figure (2): Two projections are added together where they overlap to form the summation image.

Essentially, it is known that the point of density is somewhere along that line so that a crude reconstruction results if the measured value is assigned along the entire line. Adding up the values for all $\theta$ will yield a distorted picture of the true object, sometimes called the laminogram

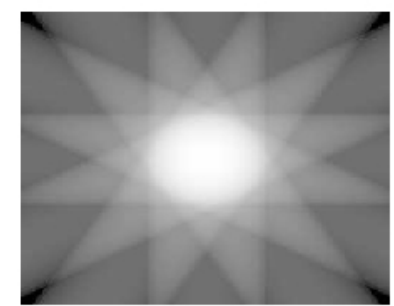

(a)

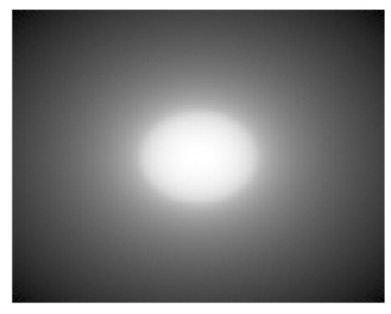

(b)

Figure (3): a)Star-shaped pattern(six projections are used) b)Blurred object(enough projections are used)

Practical results of backprojecting a circular object centred at the origin. Projections at equidistant angles are shown smeared across the 256x256 matrix and the values summed together. When only six projections are used 2.3(a), a star-shaped pattern emanating from the dense points emerges. When enough projections are used as in 2.3(b), the blurring is apparent.To see why the blurring occurs, consider an object consisting of a single point, as in Figure 4. In this case, each projection is identical. Mathematically, the back projection for a single projection of unknown density is:

$$
\mathbf{b}_{\theta}(\mathrm{x}, \mathrm{y})=\int \mathbf{p}_{\theta}(\mathbf{r}) \delta\left(\mathrm{x} \cos { }_{-}^{-}+\mathrm{y} \sin \theta-\mathbf{r}\right) \mathrm{dr}
$$

Where $\mathbf{b}_{\boldsymbol{\theta}}(\mathbf{x}, \mathbf{y})$ is the back projected density due to the projection $\mathrm{p} \theta(\mathrm{r})$. The $\delta()$ function is the Dirac delta function. If we sum over all angles, we obtain a summation image, $f_{b}(x, y)$ : 


$$
f_{b}(x, y)=\int_{0}^{\pi} b_{\theta}(x, y) d \theta
$$

We only need to sum from 0 to $\pi$ (not $2 \pi$ ) as all the information is present in this range.

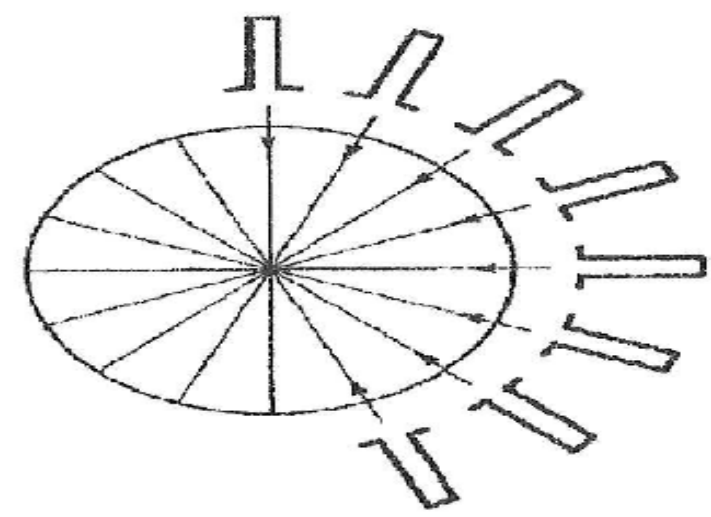

Figure (4):Projections of a point back projected are blurred by $1 / r$.

This requires interpolation from polar co-ordinates that data is measured in to the Cartesian co-ordinates of the final image.

$$
f_{b}(x, y)=f(x, z) * \frac{1}{r}
$$

The symbol ** is a $2 \mathrm{D}$ convolution. Practically, it means that the actual image, $\mathrm{f}(\mathrm{x}, \mathrm{y})$ is blurred by the $1 / \mathrm{r}$ term. In frequency space, it means that the Fourier amplitudes have been multiplied by $1 / \omega$. To get a useful image this blurring must be removed (filtered) so filtered back projection is used.

\section{A. Filtration}

It can be shown that $\mathrm{f}_{\mathrm{b}}(\mathrm{x}, \mathrm{y})$ results when each projection has been weighted by $\omega^{-1}$. The fix for this is to pre-weight the Fourier transform of each 1D projection with $|\omega|$ prior to back projecting. This is known as filtering the projections and is still the standard technique in commercial scanners. This method is efficient because it only involves a one-dimensional Fourier transform, and the FFT algorithm is used in practice

Alternatively, one may perform a space-domain convolution and dispense with Fourier transforms altogether. However this approach, which may be called a convolution-back projection, is usually more computationally intensive than using FFT's.

$|\omega|$ is what is called a ramp function, due to its shape in the frequency domain. Such a ramp filter in practice is impossible to build. It is infinite in length and it has the drawback of amplifying high frequency noise.

In practice, a variety of different filters may be used. These filters all have trade-offs among resolution and the presence of artifacts. The basic and widely used filter is the Ram-Lak filter [11]. Its conception stems from the fact that the actual measured data 
are limited to frequencies below the Nyquist frequency $\omega_{\mathrm{N}}$. The data is band-limited to $\omega_{N}$, and so the filter is too. Although ramp filter is theoretically the ideal filter to use, but real data (frequencies below $\omega_{N}$ ) contains noise and the ramp filter can enhance high frequency noise. Superior results are obtained by reducing the higher frequencies. This is performed by multiplying the $|w|$ filter by a low-pass or smoothing window that attenuates the higher frequencies which in many cases represent mainly observation noise.

Figure (5): Typical appearance of the filter multiplied by smoothing windows

$$
\begin{gathered}
\text { Ramp:(No window). shepp-logan: } \frac{\frac{\sin \left(\frac{\omega}{2 \mathbf{d}}\right)}{\left(\frac{\omega}{2 \mathbf{d}}\right)} \cdot \operatorname{Cosine}:^{\cos \frac{\omega}{2 d}} \text {. Hann: } 1+\frac{\cos \frac{\omega}{d}}{2}}{\text { Hamming: }} .54+.46 * \cos \left(\frac{\omega}{d}\right)
\end{gathered}
$$

\section{B. Interpolation}

Back-projection is the process of "smearing" the filtered projections over the reconstructing image due to the back-projection of the projection from the polar coordinates that data is measured in to the Cartesian co-ordinates of the final image. Interpolation is done and various levels of interpolation are specified. Current Interpolation Methods are:

i. Nearest Neighbor: No interpolation, selects nearest point.

ii. Linear: Uses fast straight line interpolation.

iii. Cubic: Uses cubic interpolating polynomial.

Linear Interpolation produces a visible improvement to the image with little extra processing time. Cosine, Cubic and Cubic Spline add a few extra seconds to the back projection although it is better for smooth curved edges it is not clear that there is any visible improvement in image quality. 


\section{The proposed simulation algorithm}

The proposed simulation algorithm cosists of three main stages as shown in figure 6 .

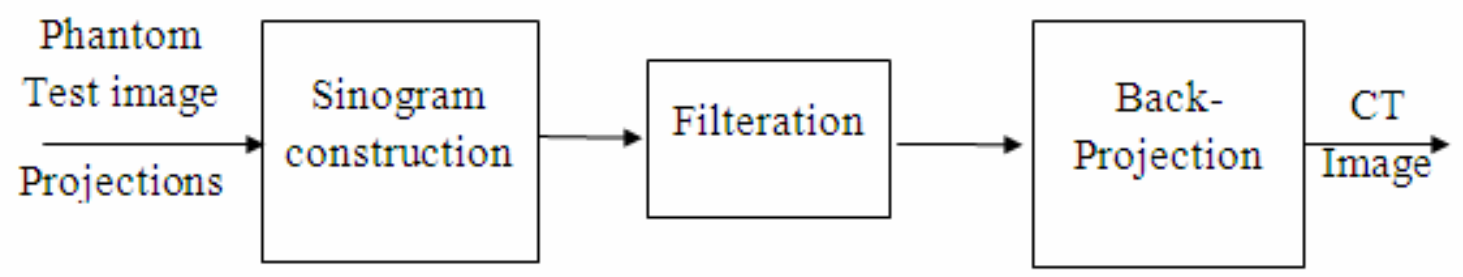

Figure (6):Algorithm stages

The following subsections will outline these three stages.

\section{A. Sinogram construction}

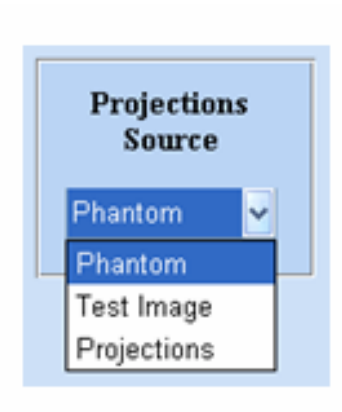

(a)

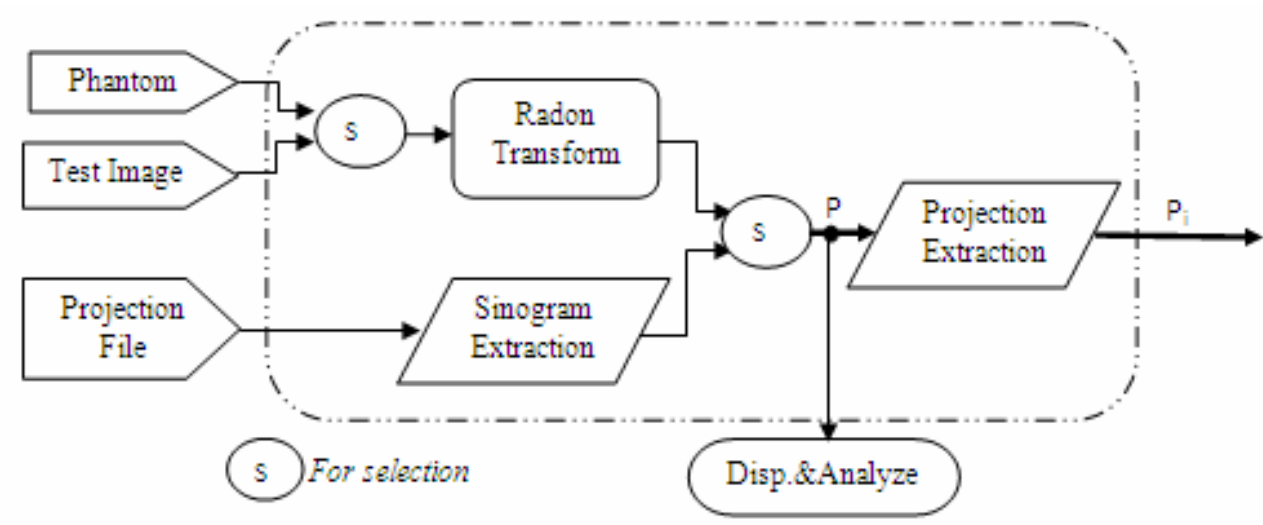

(b)

Figure (7):Projections source selection a) GUI b) Block diagram

There are three selections to get the projections data as shown in figure 7:

i) Radon transform of phantom (ready MATLAB function):- specify the phantom size and the program will simulates the process of collecting tomographic X-ray data of phantom objects.

ii) Radon transform of any test image (to be reconstructed): Load the image and the program will simulate the process of collecting tomographic Xray data.

iii) Projections (text file): Load the projection file $\left(1^{\text {st }}\right.$ row : number of detectors, $2^{\text {nd }}$ row is the number of angles, $3^{\text {rd }}$ row is the angles step and then the projections data as a matrix

The output of this stage (the sinogram) can be displayed and analyzed.

Each sinogram column (projection) is extracted to be processed separately. The output (single projection $\mathrm{P}_{\mathrm{i}}$ ) is displayed as $1^{\text {st }}$ frame. 


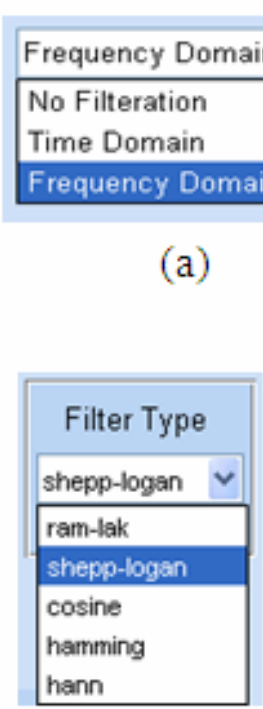

(b)

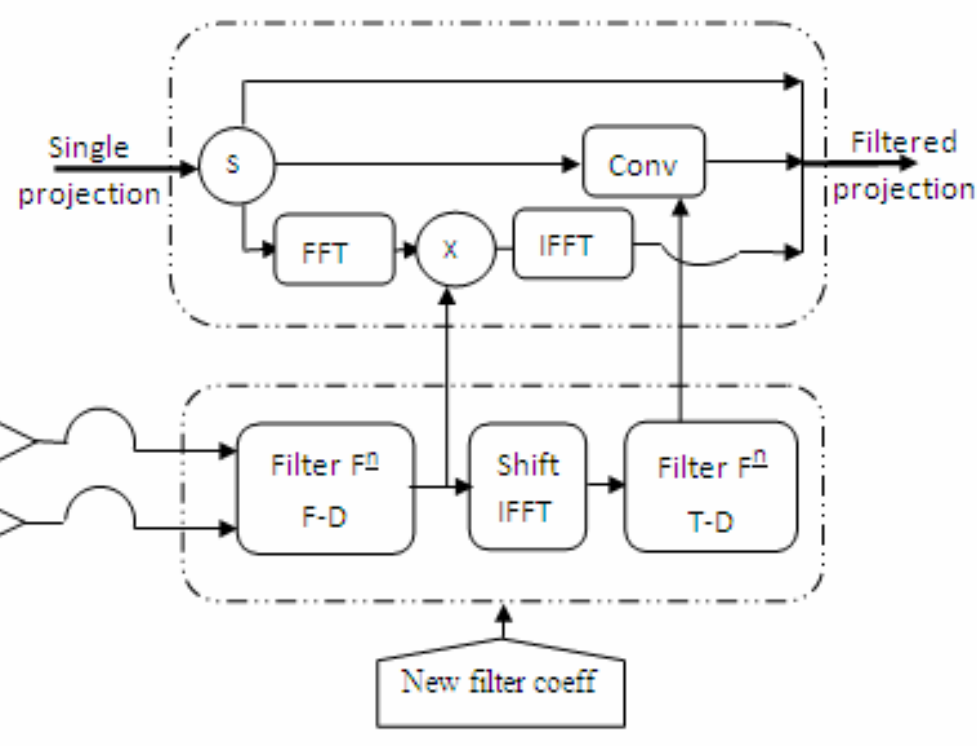

(c)

Figure (8):a) GUI filtration domain and b) Filter type c) Filtration process d) Filter formation

\section{B. Filtration}

The first step in the reconstruction process is filtering of each projection Filtration importance is discussed-The program has three selections shown in figure $8 . a \& c$

i- No filtration: - The projection is introduced to the next stage without filtering. There is an option to enter new filter coefficients

ii- Frequency domain filtering :-The FFT of the projection is multiplied with the filter function then the IFFT is performed (Filtered backprojection)

iii-Time domain filtering :- The IFFT of the filter function is shifted to be centrally localized and convoluted with the projection in the time domain (Convolution back-projection)

The window effect is also discussed and the window type selections shown in figure 8.b are Ramp filter (No window), shepp-logan, cosine, Hamming or Hann. The frequency band of the filter can be scaled with factor $\mathrm{d}$ whose range is $(.1: .1: 1)$ the scaled selected filter function is displayed in the frequency domain The output of this stage (filtered projection) can be displayed as a $2^{\text {nd }}$ frame and the filtration time is calculated (difference between $1^{\text {st }}$ and $2^{\text {nd }}$ frames) 


\section{Back Projection}

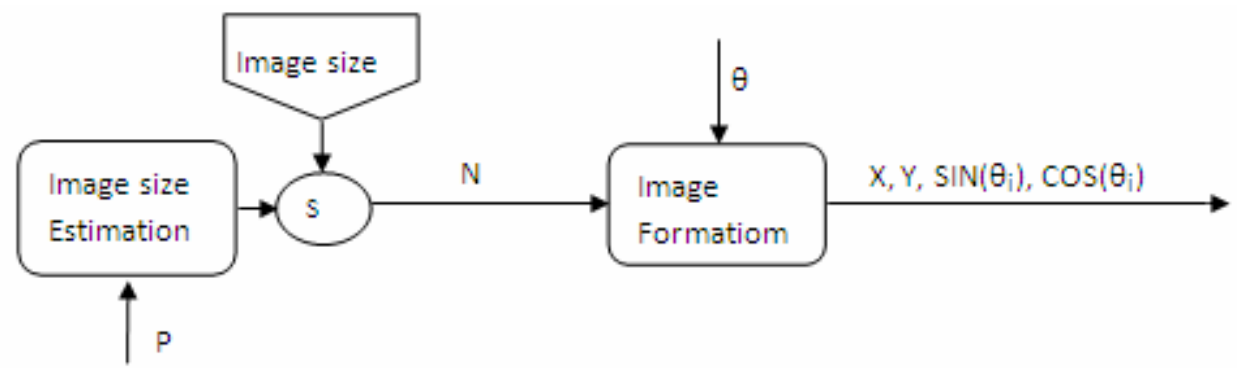

Figure (9): Image size entry and blank image formation block diagram

As shown in figure 9 the output image size $(\mathrm{N})$ is manually entered or estimated from the projection length(len), Len is equal to the image diagonal (longest projection).An empty image with this size is formed through which the filtered projection $\left(\mathrm{P}_{\mathrm{i}}\right)$ is back-projected with its corresponding angle $\left(\theta_{\mathrm{i}}\right)$. This smearing process of course need an interpolation the interpolation level is selected from Nearest, Linear or Cubic interpolation as shown in figure 10.After back-projection of each projection a new corresponding image is formed and displayed as $3^{\text {rd }}$ frame(result of smearing filtered projection through the empty image) Each new image is summated to the previous to form the final reconstructed image (sharing of all projections) this image is displayed as 4th frame the reconstruction time is calculated.

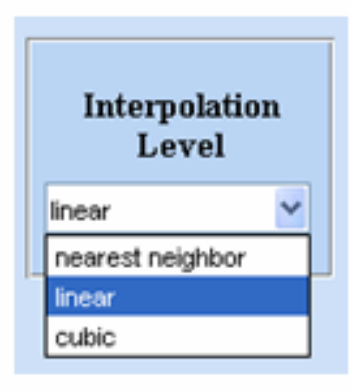

(a)

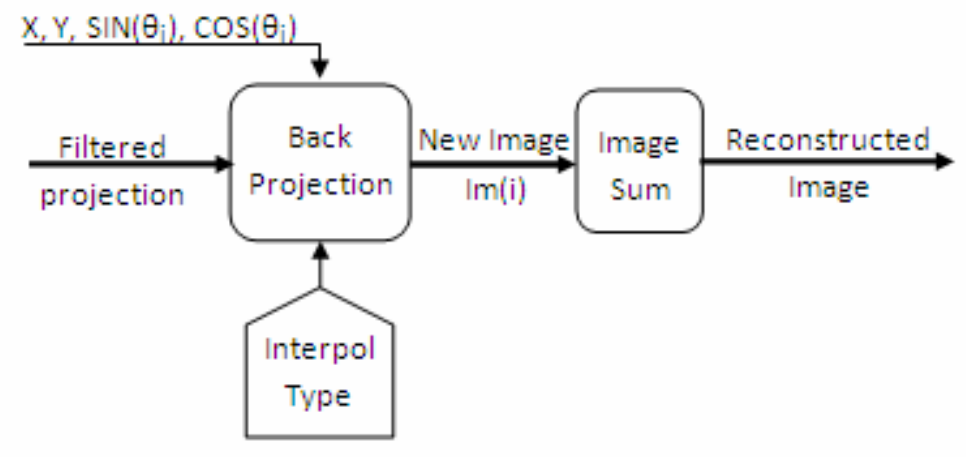

(b)

Figure (10): a) GUI interpolation level selection b) Back-projection and image summation block diagram

The GUI of the program and the block diagram of the algorithm are shown in figure 11 and 12 respectively 


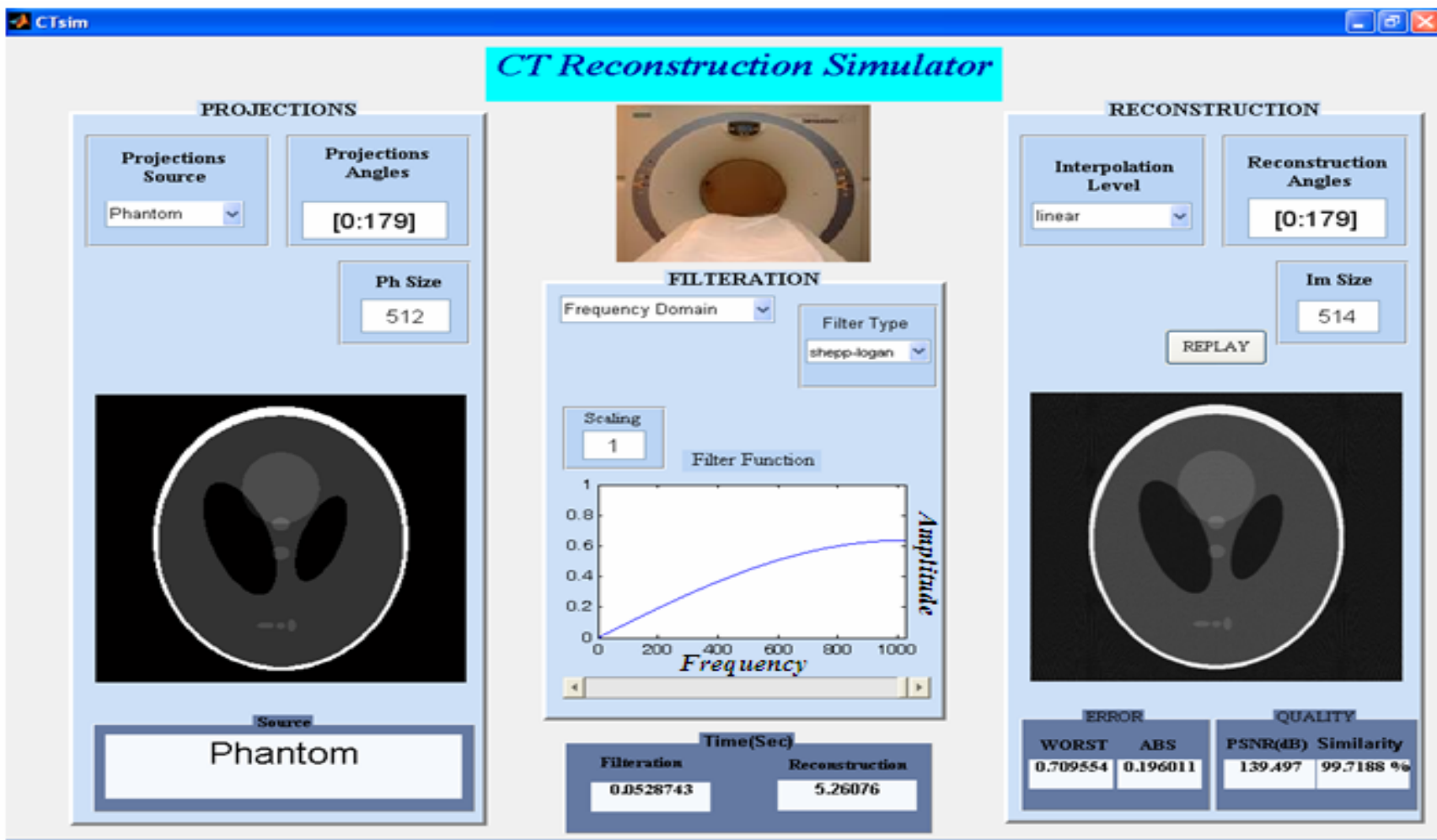

Figure (11):GUI of the program

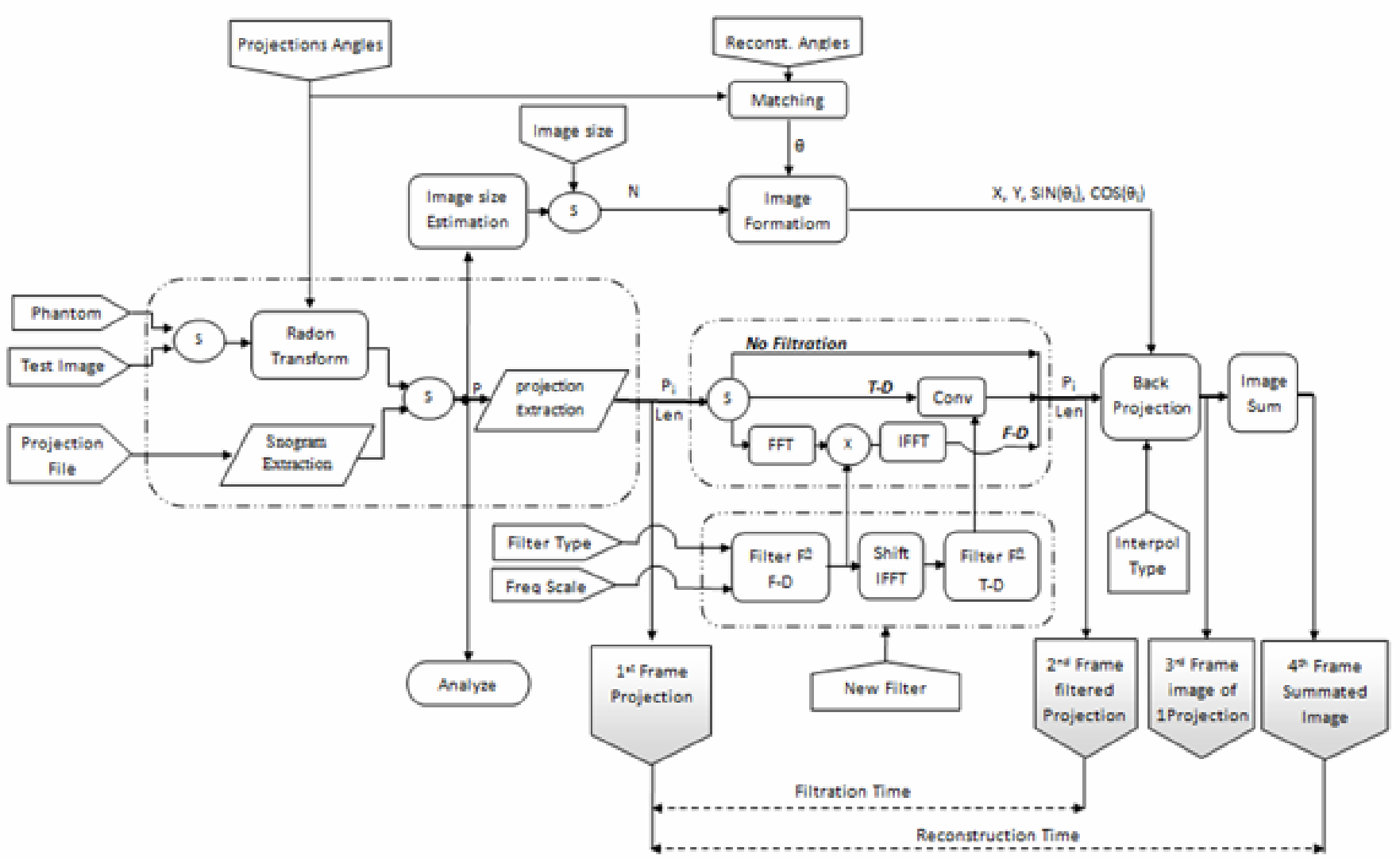

Figure (12): Block diagram of the algorithm 


\section{Visualization and analysis tools}

\section{A. Sinogram analysis}

When a projections file is loaded this analysis gives indications about some problems occur in the system during projections acquisition process

1. Detector problem: If a certain detector doesn't work probably that can be detected from its corresponding sinogram row

2. At certain angle: From the sinogram columns a problem in a certain angle shot can be detected

\section{B. Reconstruction performance Evaluation}

1. Time measurements

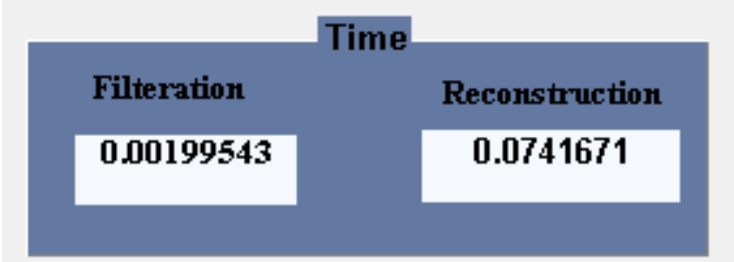

Figure (13): Time measurement display window

Beside the scan time the reconstruction time has is important to be measured, displayed and improved, the program has two time displays shown in figure 13

i- Filtration time that depends on the filtration algorithm selection and filtration domain (time or frequency)

ii-Reconstruction time including the filtration time plus the backprojection time the last depends on image size, interpolation type selection and the number of reconstruction angles

2. Reconstruction accuracy

(Case of projection source selections phantom or test image)

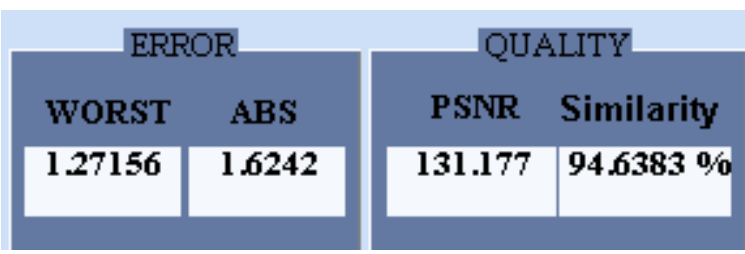

Figure (14):Reconstruction evaluation display window

Some techniques are used for reconstruction evaluation by comparing the input image (phantom or test image) and the output image (reconstructed) figure 14

i- PSNR : the peak signal to noise ratio in decibels between two images.

To compute the PSNR, first calculates the mean-squared error using the following equation: 


$$
\text { MSE }=\frac{\sum_{M, N}\left[\mathbf{I}_{1}(\mathbf{m}, \mathbf{n})-\mathbf{I}_{2}(\mathbf{m},-)\right]^{2}}{\mathbf{M} * \mathbf{N}}
$$

(8)

Where $M$ and $N$ are the number of rows and columns of input image, respectively, then computes the PSNR using the following equation:

$$
\text { PSNR }=10 \log _{10}\left(\frac{\mathbf{R}^{z}}{\text { MSE }}\right)
$$

Where $R$ is the max fluctuation in the input image (255 for 8bit)

ii- MSSIM: The mean structural similarity index between two images; if one of the images being compared is regarded as perfect quality(phantom or test image), then MSSIM can be considered as the quality measure of the other image(reconstructed) that if the measured image equal to the reference then $M S S I M=1$ or $(100 \%)$. See its mathematics details [25]

Two error measurements between the input image $\mathrm{P}$ and the output $\mathrm{R}$ are available

iii- $A B S$ : The normalized mean absolute distance measure.

$$
A B S=\frac{\sum_{i=\mathbf{1}}^{n} \sum_{j=\mathbf{1}}^{m}\left|p_{i, j}-r_{i, j}\right|}{\sum_{i=\mathbf{1}}^{n} \Sigma_{j=\mathbf{1}}^{m}\left|p_{i, j}\right|}
$$

iv-WORST: The worst case distance measure over a $2 \times 2$ pixel area.

$$
W O R S T=\max _{1 \leq k \leq[n l 2]}\left(\left|P_{k, l}-R_{k, l}\right|\right)
$$

Where

$$
\begin{aligned}
& P_{k, l}=\frac{\mathbf{1}}{\mathbf{4}}\left(p_{\mathbf{z} k, \mathbf{z} l}+p_{\mathbf{z} k+1, \mathbf{2} l}+p_{\mathbf{z} k, \mathbf{z} l+\mathbf{1}}+p_{\mathbf{z} k+1, \mathbf{z} l+\mathbf{1}}\right) \\
& R_{k, l}=\frac{1}{\mathbf{4}\left(r_{\mathbf{z} k, 2 l}+r_{\mathbf{z} k+1,2 l}+r_{\mathbf{z} k, 2 l+1}+r_{\mathbf{z} k+1,2 l+1}\right)}
\end{aligned}
$$

Note: $p$ denotes the reference image. $r$ denotes the reconstruction image. Each of the images has a size of $m \times n$. In equation (11) $[\mathrm{n} / 2]$ and $[\mathrm{m} / 2]$ denote the largest integers less than $\mathrm{n} / 2$ and $\mathrm{m} / 2$, respectively.

\section{Program displays}

\section{Filter frequency response}

The program displays the filter frequency response as shown in figure (15) with frequency band scaling factor control (Numerical or slide bar) 


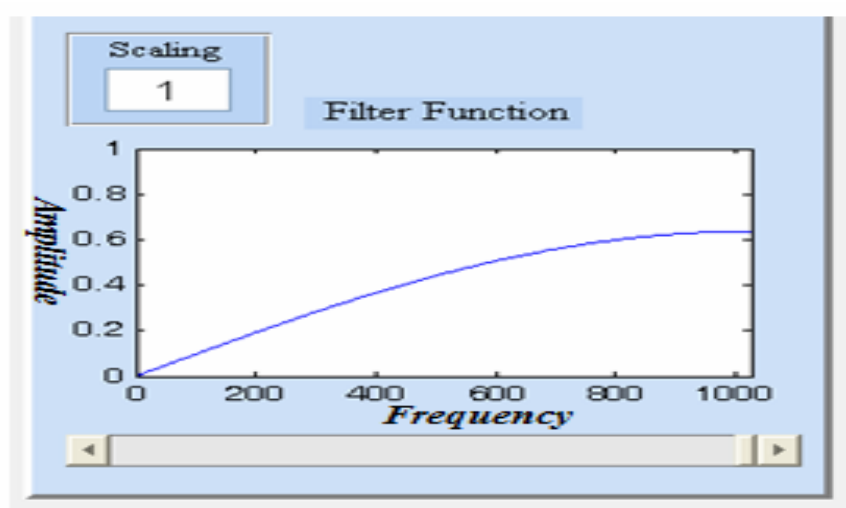

Figure (15): Filter frequency response

2. Input and output images display

The program displays both the input and output images as shown in figure 16 that make it easy to visually compare between the two image this subjective comparison should be taken into consideration as will be discussed.
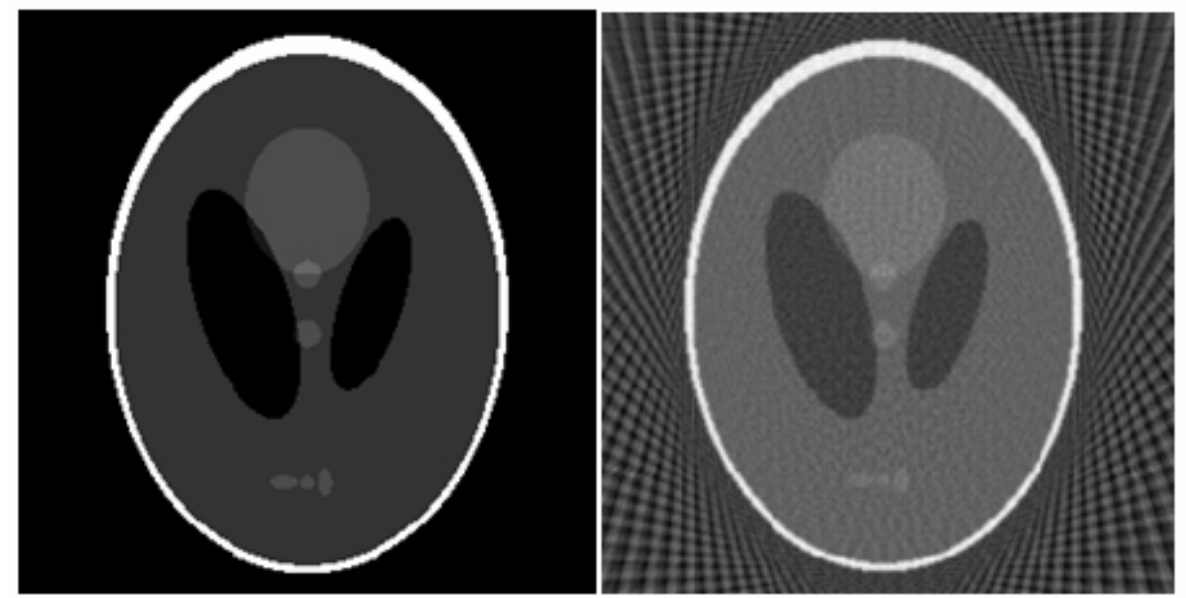

Figure (16): a)input image(phantom) b) output image(reconstructed form 45 projections)

\section{Multi-stage output display}

It has the ability to display each projection separately and the output of each stage for this projection; this option makes user the able to trace the reconstruction process visually through four frames shown figure 17 

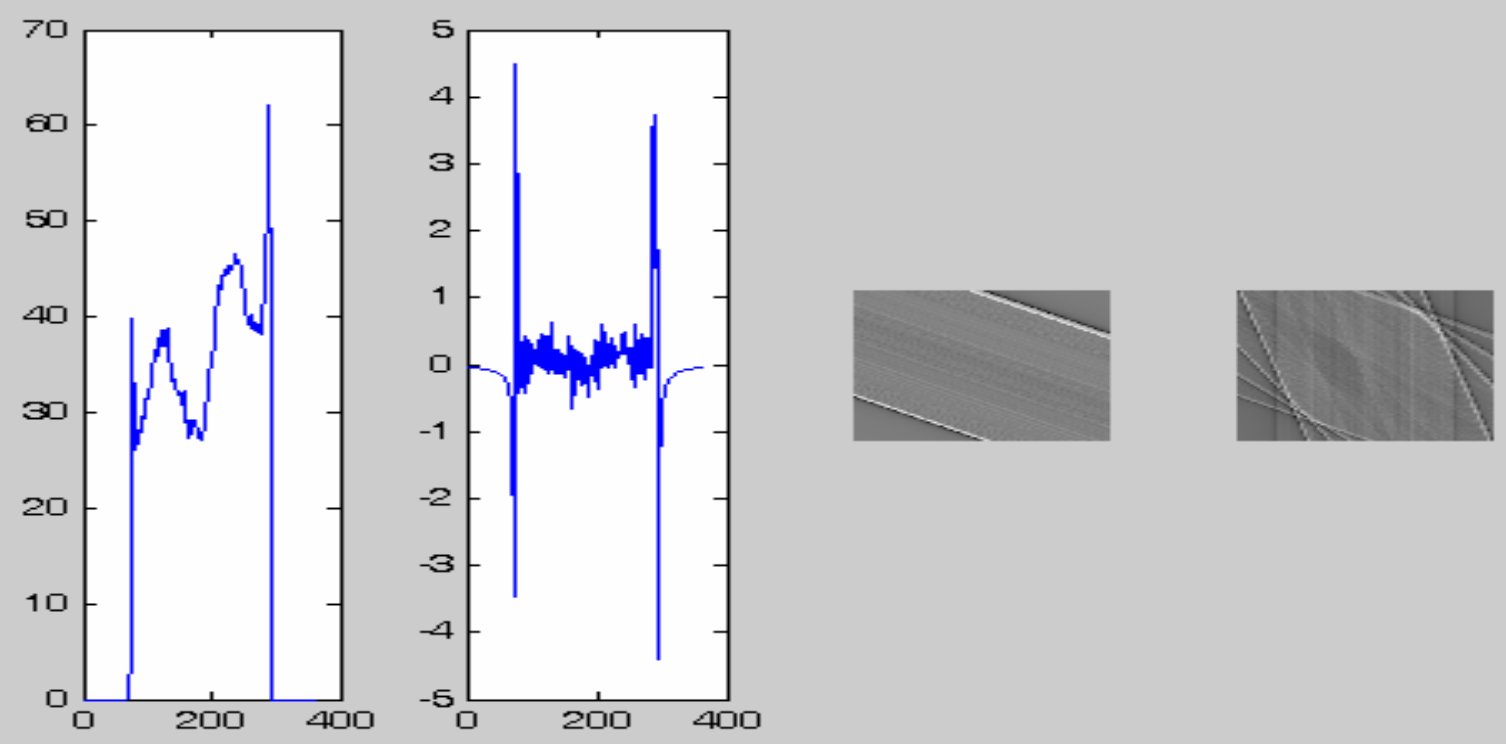

Figure (17):a) Input projection $i \quad$ b) Filtered projection c) Image from (Backprojection) projection $i$ d) Image summation from all projections $\leq i$ 


\section{$\underline{\text { 5.Conclusion and future work }}$}

This paper introduces a proposed algorithm for simulating the operation of the CT image reconstruction. This computer program is useful to study the effects of different factors on which the image quality depends. The program also produces some performance measures both objectively such as PSNR, MSSIM, Absolute error and Worst case error over $2 \times 2$ window and subjectively via multi-stage projection and image visualization. The system is user friendly because of its GUI. Also it is suitable to be used by many users with different views for image quality judgment.To show the importance and effectiveness of this program we have to ask some questions first For FBP how can we tell which filter / interpolation method to use?, then how can we decide which method gives the best resultant image?, finally how can we quantify image quality?

The answer is not trivial. It is found that no standard method exists. Everyone seems to have their own method of judging image quality! Here we find that this program gives a variety of options that enable the user to select his desired algorithm controls, trace the algorithm steps visually in addition to some algorithm evaluations

As a future work, the same basic concepts can be applied for fan-beam data. Since these earlier algorithms were proposed in the 70's and 80's, multi-slice, multi-detector scanners have become a reality. Thus three-dimensional reconstruction algorithms from cone beam data have been at the forefront of research.

Another future work to be done is parallel computer processing. Since each view can be processed independently, we can take advantage of multiple CPU's when running on a Symmetric Multiprocessing computer. Filtered backprojection is amendable to parallel processing. Indeed, this has been used in commercial scanners to speed reconstruction.

\section{$\underline{\text { References }}$}

[1] R. N. Bracewell and A. C. Riddle. Inversions of fan-beam scans in radio astronomy.

Astrophys. J., 150:427\{434, Nov 1967.

[2] G. N. Ramachandran and A. V. Lakshminarayanan. Three-dimensional Reconstruction from Radiographs and Electron Micrographs: Application of Convolutions instead of Fourier Transforms. Proc. Nat. Acad. Sci., 68:2236, 1971.

[3] Damien Farrell,Investigation and demonstration of a technique in CT image reconstruction for use with truncated data, University of Dublin, Trinity College, August 2006

[4] A. C. Kak and Malcolm Slaney. Principles of Computerized Tomographic Imaging. IEEE Press, 1988.

[5] A. Asuno. Pattern Information Processing: Topic 5. Computed Tomography Image reconstruction from projections. 2004.

[6] Edwin L. Dove. Notes on Computerized Tomography, Univ. of Iowa. 2001. 
[7] Johann Radon. ÄUber die bestimmung von funktionen durch ihre integralwerte lngs gewisser mannigfaltigkeiten, Berichte Schsische Akadamie der Wissenschaften. Math. Nat. K., 69:262\{267, 1917.

[8] G N Houns ${ }^{-}$eld. Computerized transverse axial scanning (tomography). 1. Description of system. Br J Radiol, 46(552):1016\{1022, Dec 1973.

[9] A M Cormack. Reconstruction of densities from their projections, with applications in radiological physics. Phys Med Biol, 18(2):195\{207, Mar 1973. [10]Jiang Hsieh,Computed Tomography, second edition, Society of photo-optical instrumentation engineers, 2009

[11]John C. Russ, The Image Processing Handbook, Fifth edition, North Carolina State University, 2007

[12] R. W. Hamming. Digital Filters. Englewood Cli®s, NJ: Prentice-Hall, 1977. [13] L. A. Shepp and B. F. Logan. The Fourier Reconstruction of a Head Section. IEEE Trans. Nucl. Sci., NS:21, 1974.

[14] R. W. Schafer and L. R. Rabiner. A digital signal processing approach to interpolation. Proc. IEEE, 61:697\{702, 1973.

[15] C. V. Jakowatz and A. C. Kak. Computerized Tomography using $x$-rays and ultrasound. Research Rep. TR-EE 76-26, School of Electrical Engineering, Purdue Univ., Lafayette,IN, 1976.

[16] A. V. Lakshminarayanan. Reconstruction from divergent ray data. Tech. Rep. 92, Dept.of Computer Science, State Univ. of New York at Bußalo, 1975.

[17] G. T. Herman and A. Naparstek. Fast image reconstruction based on a Radon inversion formula appropriate for rapidly collected data. SIAM. J. Appl. Math., 33:511\{533, 1977.

[18] N. Baba and K. Murata. Filtering for image reconstruction from projections. J. Opt. Soc.Amer., 67:662\{668, 1977.

[19] S. K. Kenue and J. F. Greenleaf. E \pm cient convolution kernels for computerized tomogra- phy. Ultrason. Imaging, 1:232\{244, 1979.

[20] R. M. Lewitt. Ultra-fast convolution approximation for computerized tomography. IEEE Trans. Nucl. Sci., NS(26):2678\{2681, 1979.

[21] L. Wang. Cross-section reconstruction with a fan beam scanning geometry. IEEE. Trans. Comput., C-26:351\{364, 1977.

[22] T. M. Peters and R. M. Lewitt. Computed tomography with fan beam geometry. J. Comput. Assist. Tomog., 1:429\{436, 1977.

[23] K. C. Tam. Tomographic imaging with limited angle input. J. Opt. Soc. Amer., 71:581\{592, 1981.

[24] Ge Wang, D.L. Snyder, J.A. O'Sullivan, and M.W. Vannier. Iterative deblurring for CT metal artifact reduction. IEEE Trans. Med. Imaging, 15-5:657\{664, 1996. [25] Z. Wang, A. C. Bovik, H. R. Sheikh, and E. P. Simoncelli, "Imagequality assessment: From error measurement to structural similarity"IEEE Transactios on Image Processing, vol. 13, no. 4, Apr 